\title{
Estrogen-related receptor $\gamma$ is a novel catabolic regulator of osteoarthritis pathogenesis
}

\author{
Young-Ok Son \& Jang-Soo Chun ${ }^{*}$ \\ National Creative Research Initiatives Center for Osteoarthritis Pathogenesis and School of Life Sciences, Gwangju Institute of Science and \\ Technology, Gwangju 61005, Korea
}

Osteoarthritis $(\mathrm{OA})$ is the most common form of arthritis and is a leading cause of disability with a large socioeconomic cost. $\mathrm{OA}$ is a whole-joint disease characterized by cartilage destruction, synovial inflammation, osteophyte formation, and subchondral bone sclerosis. To date, however, no effective disease-modifying therapies for OA have been developed. The estrogen-related receptors (ERRs), a family of orphan nuclear receptor transcription factors, are composed of ERR $\alpha$, ERR $\beta$, and ERR $\gamma$, which play diverse biological functions such as cellular energy metabolism. However, the role of ERRs in OA pathogenesis has not been studied yet. Among the ERR family members, $E R R \gamma$ is markedly upregulated in human and various models of mouse OA cartilage. Adenovirus-mediated overexpression of ERR $\gamma$ in the mouse knee joint tissue caused OA pathogenesis. Additionally, cartilage-specific ERR $\gamma$ transgenic (Tg) mice exhibited enhanced experimental OA. Consistently, ERR $\gamma$ in articular chondrocytes directly caused expression of matrix metalloproteinase (MMP) 3 and MMP13, which play a crucial role in cartilage destruction. In contrast, genetic ablation of Esrrg or shRNA-mediated Esrrg silencing in the joint tissues abrogated experimental $\mathrm{OA}$ in mice. These results collectively indicated that ERR $\gamma$ is a novel catabolic regulator of $O A$ pathogenesis and can be used as a therapeutic target for OA. [BMB Reports: Perspective 2018; 51(4): 165-166]

*Corresponding author. E-mail: jschun@gist.ac.kr

https://doi.org/10.5483/BMBRep.2018.51.4.019

Received 17 January 2018

Keywords: Cartilage, Estrogen-related receptor $\gamma$, Interleukin-6, Matrix metalloproteinases, Osteoarthritis

Abbreviations: ChIP, Chromatin immunoprecipitation; Coll-II, type II collagen; DMM, destabilization of medial meniscus; ECM, extracellular matrix; ER, endoplasmic reticulum; ERRs, estrogenrelated receptors; HIF-2 $\alpha$, hypoxia-inducible factor- $2 \alpha$; IL-1 $\beta$, interleukin-1 $\beta$; MMP, matrix metalloproteinase; OA, osteoarthritis

Perspective to: Son YO, et al. (2017) Estrogen-related receptor $\gamma$ causes osteoarthritis by upregulating extracellular matrix-degrading enzymes, Nat Commun, 8(1):2133. doi: 10.1038/s41467-017-018688
Osteoarthritis (OA) is caused by an imbalance between anabolic and catabolic factors which is induced by a variety of etiologic risk factors and pathophysiological processes. Important among potential OA-causing mechanisms are mechanical stresses, including joint instability and injury, and factors that predispose individuals toward $\mathrm{OA}$, such as aging. These factors alter biochemical pathways in chondrocytes, resulting in degradation of the extracellular matrix (ECM) by matrix metalloproteinases (MMPs) and aggrecanases (ADAMTSs). Among the matrix-degrading enzymes, MMP3, MMP13, and ADAMTS5 are known to play crucial roles in OA cartilage destruction. Various catabolic regulators such as a proinflammatory cytokine, and interleukin (IL)-1 $\beta$, cause upregulation of MMP3, MMP13, and ADAMTS5.

The ERRs are known to regulate a variety of isoform-specific biological functions under normal and pathophysiological conditions. However, the functions of ERRs in the regulation of joint cartilage homeostasis and/or OA pathogenesis has not been studied yet. To explore possible association of ERRs with OA pathogenesis, the expression levels of ERRs in OA cartilage of human and various mouse models were examined. mRNA levels of all ERR isoforms (ERR $\alpha$, ERR $\beta$, and ERR $\gamma$ ) were significantly elevated in OA-affected damaged regions of human cartilage compared with undamaged regions of arthritic cartilage. However, only ERR $\gamma$, but not ERR $\alpha$ and $E R R \beta$, was significantly increased in experimental mouse OA cartilage caused by the destabilization of the medial meniscus (DMM), which most likely mimics human OA. Consistent with increased mRNA levels, immunostaining revealed that ERR $\gamma$ protein levels were also markedly elevated in chondrocytes of both human and DMM-induced mouse OA cartilages. ERR $\gamma$ is upregulated by multiple signaling pathways, including alcoholinduced activation of the cannabinoid receptor, IL-6-mediated inflammation, fasting-induced glucagon receptor activation, feeding-induced insulin receptor activation, hypoxia, and ER (endoplasmic reticulum) stress (Misra J, Kim DK, Choi HS (2017) Trend Endocrinol Metab 28, 261-272). Among the upstream regulators of ERR $\gamma$, IL-6 was specifically increased by IL-1 $\beta$, hypoxia-inducible factor (HIF)- $2 \alpha$, and ZIP8 and IL-6 treatment in primary culture chondrocytes caused upregulation of ERR $\gamma$, indicating a role of IL-6 in ERR $\gamma$ expression. 


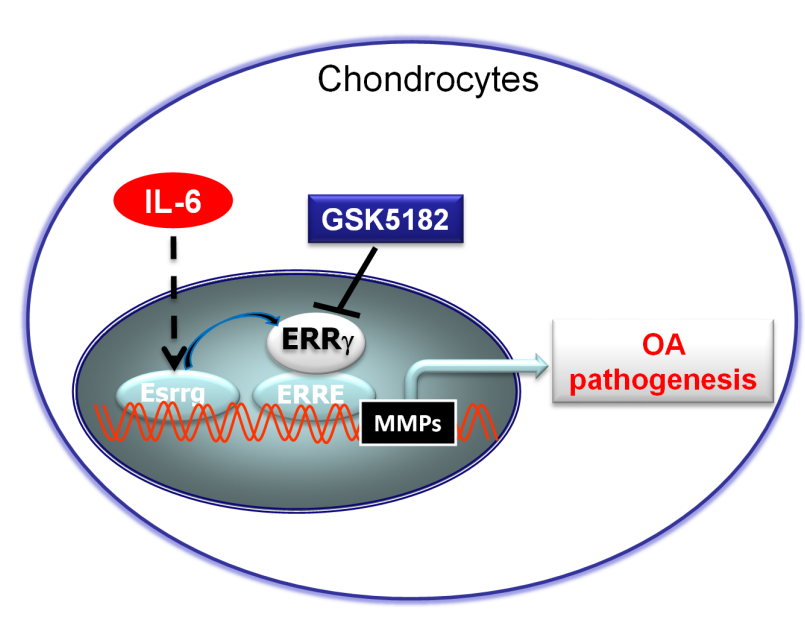

Diagram 1. Proposed model of IL-6-mediated ERR $\gamma$ functions as a catabolic regulator of OA pathogenesis.

Ectopic expression of ERR $\gamma$ in the mouse joint tissues via IA injection of adenovirus expressing ERR (Ad-Esrrg) (3-weeks) caused cartilage destruction, as determined by safranin $O$ staining and synovial inflammation, as determined by $\mathrm{H} \& \mathrm{E}$ staining. Cartilage destruction was more severe at 8 weeks after IA injection of Ad-Esrrg. Other manifestations of OA, including osteophyte formation and subchondral bone sclerosis, were also observed at 8 weeks after IA injection of Ad-Esrrg. The role of ERR $\gamma$ in OA pathogenesis was further validated by generating cartilage-specific ERR $\gamma \mathrm{Tg}$ mice by using a Col2a1 enhancer and promoter. Col2a1-Esrrg Tg mice showed increased mRNA and protein levels of ERR $\gamma$ in cartilage tissue without defects in skeletal development. Compared with wild-type (WT) littermates, Col2a1-Esrrg Tg mice exhibited significantly enhanced cartilage destruction, subchondral bone sclerosis, and osteophyte formation upon DMM surgery. Thus, these gain-of-function studies collectively indicate that ERR $\gamma$ is a catabolic regulator of OA pathogenesis. For the loss-of-function studies, ERR $\gamma$ knock-out (KO) or Ad-shEsrrg IA-injected mice were used for DMM surgery. OA manifestations caused by DMM surgery, including cartilage erosion, osteophyte formation, and subchondral bone sclerosis, were significantly suppressed in $\mathrm{Esrrg}^{+/-}$mice compared with WT littermates. Consistently, silencing Esrrg in the knee joint tissues via IA injection of Ad-shEsrrg also significantly suppressed cartilage erosion, subchondral bone sclerosis, and osteophyte formation in DMM-operated mice. These results collectively indicate that knockdown or genetic ablation of Esrrg in mice inhibits OA pathogenesis caused by DMM surgery.

Overexpression of ERR $\gamma$ in chondrocytes via infection of Ad-Esrrg caused upregulation of MMP3 and MMP13, without affecting ADAMTS4 or -5 . In contrast, mRNA levels of SOX9, type II collagen (Coll-II), and aggrecan were reduced by overexpression of ERR $\gamma$, although the degree of inhibition was small. Consistent with the increased mRNA levels, protein levels of MMP3 and MMP13 were also markedly elevated in chondrocytes infected with Ad-Esrrg. Consistent with these findings, MMP3 and MMP13 proteins were upregulated in Ad-Esrrg-injected cartilage tissue, whereas SOX9 and type II collagen protein levels were markedly reduced. Chromatin immunoprecipitation (ChIP) assays demonstrated that ERR $\gamma$ regulates Mmp3 and Mmp13 in chondrocytes as direct target genes. Furthermore, treatment with the ERR $\gamma$ inverse agonist GSK5182 inhibited IL-6-induced ERR $\gamma$, MMP3, and MMP13 upregulation in chondrocytes (Diagram 1). Also, intraperitoneal administration of GSK5182 reduced DMM-induced OA pathogenesis. Collectively, these results are the first to demonstrate that ERR $\gamma$ acts as a catabolic regulator of cartilage degeneration and OA pathogenesis, and support the idea that ERR $\gamma$ could be a therapeutic target for OA.

\section{ACKNOWLEDGEMENTS}

This work was supported by grants from the National Research Foundation of Korea (2016R1A3B1906090 and 2016R1A5A10 07318), the Korea Health Technology R\&D Project through the Korea Health Industry Development Institute (HI16C0287 and HI14C3484), and the GIST Research Institute (GRI) in 2017. Y.-O.S. was supported by a grant from the Basic Science Research Program through the National Research Foundation of Korea (2016R1D1A1B03930327). 